\title{
Variación altitudinal de los atributos morfo-fisiológicos en dos especies de plantas alto-andinas y sus implicancias contra la fotoinhibición
}

\author{
Altitudinal variation of morpho-physiological traits in two High-Andean plant species \\ and its effects against the photoinhibition
}

\author{
Marco A. Molina-Montenegro ${ }^{1,2,3}$ \& Lohengrin A. Cavieres ${ }^{2,3}$ \\ ${ }^{1}$ Centro de Estudios Avanzados en Zonas Áridas (CEAZA), Facultad de Ciencias del Mar, Universidad Católica \\ del Norte. Larrondo 1281, Coquimbo, Chile. \\ 2Departamento de Botánica, Universidad de Concepción, Concepción, Chile. \\ ${ }^{3}$ Instituto de Ecología y Biodiversidad (IEB), Universidad de Chile, Santiago, Chile. \\ marcmoli@udec.cl
}

\begin{abstract}
RESUMEN
Los ambientes de alta-montaña se caracterizan por presentar condiciones climáticas adversas para el desarrollo de las plantas. Dentro de estas condiciones climáticas destacan las bajas temperaturas del aire y del sustrato, la alta radiación lumínica y el déficit hídrico. Se ha documentado que la alta radiación lumínica y las bajas temperaturas típicas de la alta-montaña serían propicias para la fotoinhibición. No obstante, existen muchas plantas que pueden distribuirse de manera exitosa sobre gradientes altitudinales, sugiriendo la presencia de estrategias para lidiar con las condiciones climáticas adversas. En el presente estudio se evaluaron diferentes estrategias fotoprotectoras tanto morfológicas como fisiológicas que poseen las especies Taraxacum officinale G.Weber ex F.H.Wigg. (Asteraceae) y Phacelia secunda F.J.Gmel. (Hydrophyllaceae) creciendo en dos poblaciones. Se midieron los atributos morfológicos y fisiológicos en individuos de ambas especies creciendo a los 2.600 y 3.600 m, en Los Andes de Chile central. Los resultados muestran que en T. officinale variaron cuatro de los seis atributos morfológicos medidos entre las dos altitudes, no obstante $P$. secunda presentó variación con la altitud en todos sus atributos morfológicos. Por otro lado, los individuos de T. officinale que crecen a mayor altitud presentaron significativamente una mayor concentración de pigmentos implicados en el ciclo de las xantófilas, mientras que $P$. secunda no presentó diferencias, sugiriendo que $T$. officinale tiene una mayor capacidad para disipar la energía solar como calor. En ambas especies, el estado de desepoxidación fue mayor en aquellos individuos provenientes de la población de mayor altitud. Los resultados sugieren que mientras $T$. officinale posee una estrategia principalmente fisiológica para lidiar con las condiciones fotoinhibitorias de la alta-montaña, $P$. secunda posee una estrategia principalmente morfológica. No obstante estas estrategias más que ser excluyentes parecen ser complementarias. De esta manera, la presencia de una determinada especie en la zona de la alta-montaña dependerá en gran medida de las estrategias particulares que posea cada una de ellas para evitar la fotoinhibición por alta radiación y bajas temperaturas típica de las zonas de la alta-montaña de Chile central.
\end{abstract}

Palabras clave: Ángulos foliares, bajas temperaturas, ciclo de las xantófilas, foto-protección, Los Andes.

\begin{abstract}
High-mountain environments are characterized by stressful climatic conditions for the development of plants. Some of these conditions are low temperatures of air and soil, high radiation, and water shortage. It has been documented that high radiation and low temperatures are favorable conditions for inducing photoinhibition. Nevertheless, there are plant species that successfully live on extended altitudinal gradients, suggesting the presence of strategies to cope with stressful climatic conditions. In this study we assessed the different photoprotective strategies, both morphological and physiological in Taraxacum officinale G.Weber ex F.H.Wigg. (Asteraceae) and Phacelia secunda F.J.Gmel. (Hydrophyllaceae) growing in two populations. Physiological and morphological traits were recorded in both species growing at 2,600 y 3,600 m, in Los Andes of Central Chile. Overall, results indicate that $T$. officinale showed variations in four of the six morphological traits recorded between altitudes; however, $P$. secunda showed high morphological variations among populations in all of its traits. On the other hand, T. officinale individuals growing in the upper population significantly showed a higher amount of pigments involved in xanthophyll cycle, while $P$. secunda showed no differences, suggesting that $T$. officinale has most capacity for dissipation of the solar energy as heat. In both species, the desepoxidation status was significantly higher in those individuals from upper populations. Results suggest that while T. officinale has mainly a physiological
\end{abstract}


Gayana Bot. 67(1), 2010

strategy to cope with the photoinhibitory conditions in the high-mountain, $P$. secunda has a strategy mainly based in morphological variations. Therefore, the presence of specific plant species in the high-mountain habitats should be related with the different strategies for avoiding fotoinhibition by high radiation and low temperature, which are typical from the high-mountain habitats of Central Chile.

KEYwords: Foliar angles, low temperature, xanthophyll cycle, photo-protection, Los Andes.

\section{INTRODUCCIÓN}

La alta radiación combinada con bajas temperaturas ha sido sugerida como una condición propicia para la fotoinhibición debido a un desbalance energético (Demming-Adams \& Adams 1996, Huner et al. 1998, Streb et al. 2003a). La fotoinhibición es definida como la disminución en el desempeño fotosintético debido a un exceso de energía lumínica absorbida (Long et al. 1994). Debido al desacople entre procesos biofísicos y bioquímicos, las plantas captarían más energía de la que pueden utilizar fotosintéticamente, provocando este desbalance energético (Osmond 1994, Huner et al. 1998, Niyogi 1999, 2000).

Los ambientes de alta-montaña se caracterizan por presentar una alta rigurosidad climática, donde destacan las bajas temperaturas del aire y del sustrato, déficit hídrico, alta radiación y escasez de nutrientes (Körner 2003). Estas características hacen que los ambientes de alta-montaña sean muy estresantes y propicios para un desbalance energético, provocando fotoinhibición y/o fotodaño en las plantas (Wildi \& Lütz 1996, Streb et al. 1998). Por ejemplo, Germino \& Smith (2000) mostraron que las especies alto-andinas Caltha leptosepala DC. (Ranunculaceae) y Erythronium grandiflorum Pursh (Liliaceae), evidencian limitación en el desempeño fisiológico al ser expuestas a altas radiaciones y bajas temperaturas. En ambas especies se observó una disminución de la eficiencia fotoquímica potencial del fotosistema II y un aumento de la disipación de energía. De esta manera, se sugiere que las condiciones de alta radiación y baja temperatura afectarían de manera negativa el desempeño fotosintético, incluso para especies que habitan las zonas de alta-montaña.

Por otro lado, algunos estudios han demostrado que las especies que crecen en zonas de alta-montaña estarían adaptadas a las altas intensidades lumínicas y bajas temperaturas características de estos ambientes, no evidenciando signos de fotoinhibición. Por ejemplo, Pittermann \& Sage (2000) mostraron que la especie Bouteloua gracilis (Kunth) Lag. ex Griffiths (Poaceae) creciendo a los 1.500 y 3.000 m en las Montañas Rocallosas, posee valores de eficiencia fotoquímica $(\mathrm{Fv} / \mathrm{Fm})$ de $0,74 \mathrm{y}$ 0,76 respectivamente. Por lo anterior, se sugiere que mayor radiación y menores temperaturas no necesariamente tienden a una disminución en el desempeño fisiológico, debido a que valores cercanos a 0,75 son indicadores de un buen estado fisiológico por parte de la planta (Maxwell \& Johnson 2000). Adicionalmente, Streb et al. (2003b) demostraron que plantas de alta-montaña presentan tasas fotosintéticas similares a individuos de la misma especie de menores altitudes, sugiriendo que plantas alpinas presentarían diversos mecanismos o estrategias para lidiar con las condiciones adversas típicas de estos ambientes (Streb et al. 1997, Körner 2003).

Se ha documentado que muchas especies vegetales que habitan las zonas de alta-montaña poseen mecanismos y estrategias efectivas para evitar las condiciones abióticas que provocan el desbalance energético en estos ambientes (Körner \& Larcher 1988, Streb et al. 1997, 2003a). Dentro de estas estrategias destacan las de tipo morfológicas: variación en el tamaño del individuo, variación del área foliar específica, presencia de pubescencia, mayor auto-sombreamiento y variación en los ángulos foliares (Muraoka et al. 1998, Galen 2006, Meinzer et al. 1985, Ikeda \& Matsuda 2002, Molina-Montenegro 2008); entre las estrategias de tipo fisiológicas se encuentran: disipación térmica mediante el ciclo de las xantófilas, fotorrespiración y la disminución en la razón clorofila/pigmentos protectores (Demming-Adams \& Adams 1996, Caasi-Lit et al. 1997, Manuel et al. 1999, Niyogi 2000, Öncel et al. 2004).

En Los Andes de Chile central a medida que se asciende en altitud se registra un aumento en la radiación incidente y una disminución en la temperatura del aire, tornándose un ambiente propicio para la fotoinhibición. A pesar de lo anterior, es posible encontrar algunas especies creciendo sobre amplios rangos de distribución altitudinal. Dentro de estas especies destacan Phacelia secunda J.F.Gmel. (Hydrophyllaceae) y Taraxacum officinale G.Weber ex F.H.Wigg. (Asteraceae) las cuales presentan una extraordinaria amplitud creciendo desde el nivel del mar hasta los límites de distribución vegetacional en la zona de Los Andes de Chile central (3600 m) (Cavieres 2000, Cavieres et al. 2008). Si bien, a medida que se aumenta en altitud el ambiente se torna más limitante para la mantención de un buen desempeño fisiológico, $P$. secunda y $T$. officinale que crecen en distintas poblaciones sobre este gradiente altitudinal no evidencian signos de fotoinhibición, manteniendo una alta eficiencia fotoquímica del fotosistema II en todas las altitudes (Fig. 1). 
Por lo expuesto anteriormente, se sugiere que las plantas presentes sobre gradientes altitudinales en las zonas de alta-montaña debiesen presentar estrategias morfológicas, fisiológicas o ambas para evitar un desbalance energético, disminuyendo el riesgo de fotoinhibición. Adicionalmente, estas estrategias debiesen tener mayor relevancia en individuos creciendo a mayores elevaciones, donde las condiciones ambientales son más propicias para la fotoinhibición. En el presente estudio se evaluó las estrategias morfológicas y fisiológicas contra el riesgo de fotoinhibición en individuos de $P$. secunda y $T$. officinale creciendo en dos poblaciones a los 2.600 y $3.600 \mathrm{~m}$, en Los Andes de Chile central.

\section{MATERIALES Y MÉTODOS}

\section{Sitio DE ESTUdiO}

El presente estudio fue realizado en Los Andes de Chile central a $70 \mathrm{~km}$ al este de la ciudad de Santiago. En esta área se seleccionaron dos poblaciones de $P$. secunda y T. officinale a diferentes elevaciones $(2.600$ y $3.600 \mathrm{~m})$ y con la misma exposición. El sitio de menor elevación $(2.600 \mathrm{~m})$ se encuentra sobre una ladera de exposición oeste; la vegetación corresponde al límite superior del matorral sub-andino, caracterizada por la dominancia del arbusto Chuquiraga oppositifolia D.Don (Asteraceae) y especies del género Acaena (Cavieres 2000). El sitio de mayor elevación $(3.600 \mathrm{~m})$ se localiza en la cima del cerro Franciscano. Este sitio corresponde al piso andino superior, donde la vegetación es dominada por la planta en cojín Azorella monantha Clos (Apiaceae) y especies herbáceas de bajo tamaño como Nassauvia pyramidalis Meyen (Asteraceae) y Pozoa coriacea Lag. (Apiaceae) (MolinaMontenegro et al. 2009).

Las precipitaciones en Los Andes de Chile central ocurren principalmente como nieve y durante los meses de invierno; el verano es seco, con ocasionales lluvias principalmente a altas elevaciones. La precipitación anual sobre el límite arbóreo $(2.100 \mathrm{~m})$ ha sido estimada entre 400-900 mm (Santibáñez \& Uribe 1990). A los 3.600 m, si bien las precipitaciones debiesen ser mayores, no se tienen registros. La temperatura media anual del aire para la estación de crecimiento a los 2.600 y $3.600 \mathrm{~m}$, ha sido estimada en $10,2^{\circ} \mathrm{C}$ y $3,4{ }^{\circ} \mathrm{C}$, respectivamente (Cavieres \& Arroyo 1999). La radiación fotosintéticamente activa (PAR) en el mes de febrero alcanza valores máximos promedios de 1.910 y $2.490 \mu \mathrm{mol} \mathrm{m} \mathrm{m}^{-2} \mathrm{~s}^{-1}$ para $\operatorname{los} 2.600$ y $3.600 \mathrm{~m}$, respectivamente.

\section{ESPECIES DE ESTUDIO}

Taraxacum officinale es una hierba perenne distribuida alrededor de todo el mundo, que crece en gradientes latitudinales y altitudinales (Cavieres et al. 2005). En Chile se encuentra desde la Región de Antofagasta hasta Tierra del Fuego (Zuloaga et al. 2008). En un gradiente altitudinal, esta especie se ha observado desde el nivel del mar hasta los 3.700 m, en Los Andes de Chile central (obs. pers.). Si bien, a medida que se aumenta en altitud las condiciones abióticas se tornan más limitantes, esta especie presenta características morfológicas que no difieren de gran manera entre las distintas altitudes (Molina-Montenegro et al. 2009).

Phacelia secunda es una hierba perenne que habita en todo el continente americano, desde el sur de los Estados Unidos hasta el extremo sur de Sudamérica (Deginani 1982). En Chile, esta especie presenta una extraordinaria amplitud altitudinal, habitando desde el nivel del mar hasta los $3600 \mathrm{~m}$, y a diferencia de otras especies con amplia distribución, se desarrolla exitosamente en climas muy diversos (Cavieres et al. 2000). Sin embargo, es escasa la información que se tiene sobre la fisiología de esta especie y los mecanismos que le permiten desarrollarse con éxito a través de las condiciones abióticas limitantes que se presentan a lo largo de gradientes altitudinales. Antecedentes previos han demostrado que esta especie presenta variación en sus atributos morfológicos con la altitud (Cavieres 2000). Un estudio preliminar demostró que la presencia de pubescencia foliar le conferiría una gran protección contra la fotoinhibición en condiciones de alta radiación y baja temperatura (Molina-Montenegro, datos no publicados), lo que sugiere que el poseer diversos atributos morfológicos actuarían como estrategias fotoprotectoras en esta especie.

\section{AtributOS MORFOLÓGICOS}

A inicio de la estación de crecimiento (noviembrediciembre) fueron seleccionados 20 individuos de cada especie con tamaños similares y visualmente sanos, tanto a los 2.600 como a los $3.600 \mathrm{~m}$. Mediante una huincha métrica se registró el largo de los ejes principales de cada individuo. A partir de estos valores se calculó el tamaño de cada individuo mediante la estimación del área de una elipse. Adicionalmente se cuantificó el número de hojas y la densidad foliar de cada individuo $\left(\mathrm{N}^{o}\right.$ de hojas $\left./ \mathrm{m}^{2}\right)$. Mediante un programa de análisis de imágenes (Sigma-Scan Pro 5.0) se calculó el área foliar en tres hojas por cada individuo y en cada altitud. Las hojas utilizadas para cuantificar el área foliar fueron depositadas en una estufa de secado por $72 \mathrm{~h}$ a $65{ }^{\circ} \mathrm{C}$ para obtener el peso seco de cada hoja, pudiendo de esta manera calcular el área foliar específica (área/biomasa) de cada individuo. A partir de los valores obtenidos por el programa de análisis de imágenes se calculó la forma de cada hoja $\left(=[4 \pi x\right.$ área $] /$ [perímetro] $\left.{ }^{2}\right)$. Este índice permite medir el grado de circularidad de un objeto bidimensional, obteniéndose valores cercanos a cero en objetos lineares y cercanos a uno en objetos circulares.

Por otro lado, se midieron los ángulos foliares de cinco hojas totalmente expuestas al sol en individuos de $T$. 
officinale y de $P$. secunda en ambas altitudes mediante un transportador graduado. En cada uno de los individuos de $P$. secunda utilizados para medir el ángulo foliar se extrajeron tres hojas, las cuales fueron llevadas al laboratorio para cuantificar el largo y la densidad de la pubescencia foliar, mediante una lupa graduada de disección (Olympus TL2). Los individuos de $T$. officinale no presentaron pubescencia foliar evidente, por ese motivo estas estructuras sólo fueron medidas en $P$. secunda.

\section{Pigmentos Fotoprotectores}

En siete individuos de cada especie y por cada altitud se extrajeron cinco hojas totalmente expuestas al sol y colectadas a mediodía para estimar la concentración de pigmentos foliares implicados en el ciclo de las xantófilas. El tejido de $T$. officinale y $P$. secunda se transportó en nitrógeno líquido hasta el laboratorio para posteriormente ser analizado. El tejido congelado fue mezclado con $\mathrm{CaCO}_{3}$ y fue molido en acetona al $100 \%$ a $4{ }^{\circ} \mathrm{C}$. La cuantificación de los pigmentos implicados en el ciclo de las xantófilas: violaxantina $(\mathrm{V})$, anteraxantina $(\mathrm{A})$ y zeaxantina $(\mathrm{Z})$, se realizó mediante análisis de cromatografía "HPLC" (ver, Pérez-Torres et al. 2004). La cuantificación se realizó comparando las concentraciones absolutas $(\mathrm{V}+\mathrm{A}+\mathrm{Z})$ de las diferentes muestras recolectadas con estándares puros. Una vez obtenidas las concentraciones de los diferentes pigmentos implicados en el ciclo de las xantófilas se pudo cuantificar el estado de desepoxidación $(=\mathrm{Z}+(0,5 \times \mathrm{A}) /$ $(\mathrm{V}+\mathrm{A}+\mathrm{Z}))$, lo cual nos da cuenta del grado de disipación del exceso de energía mediante la transformación de anteraxantina hasta zeaxantina (Gilmore 1997).

AnÁLISIS ESTADÍSTICO

Los diferentes atributos morfológicos así como la concentración absoluta de pigmentos foto-protectores y el estado de desepoxidación fueron analizados mediante un análisis de varianza (ANOVA) de una vía, considerando la altitud como variable independiente. Previamente se evaluó la normalidad de los datos y la homogeneidad de la varianza mediante los test Shapiro-Wilks y Bartlett, respectivamente (Sokal \& Rohlf 1995). Todas las comparaciones altitudinales se realizaron de manera independiente para $T$. officinale y $P$. secunda.

\section{RESULTADOS}

Morfología

Los individuos de Taraxacum officinale, creciendo en el piso altitudinal superior $(3.600 \mathrm{~m})$, fueron significativamente de mayor tamaño y sus hojas presentaron un índice de circularidad mayor, en comparación con aquellos provenientes de los $2.600 \mathrm{~m}$ (Fig. 2, Tabla I). Aquéllos individuos provenientes del piso vegetacional inferior presentaron una mayor densidad foliar en comparación con aquellos de los $3.600 \mathrm{~m}$. Por otro lado, el número de hojas promedio por individuo y el área foliar específica no fueron diferentes entre los individuos provenientes de ambas altitudes (Fig. 2, Tabla I).

TABLA I. Resultados del análisis de varianza (ANDEVA) para los diferentes atributos morfológicos medidos en individuos de Taraxacum officinale y Phacelia secunda creciendo a $\operatorname{los} 2.600$ y $3.600 \mathrm{~m}$, en Los Andes de Chile central. Valores significativos se muestran en negrita.

TABLE I. Results of analysis of variance (ANOVA) for different morphological traits measured in individuals of Taraxacum officinale and Phacelia secunda growing at 2,600 and 3,600 m, in Los Andes of central Chile. Significant values are highlighted in bold.

\begin{tabular}{lcccccc}
\hline \multirow{2}{*}{ Aтributo } & \multicolumn{3}{c}{ Taraxacum officinale } & \multicolumn{3}{c}{ Phacelia secunda } \\
\cline { 2 - 7 } & g. 1. & $\mathrm{F}$ & $P$ & $\mathrm{~g} .1$. & $\mathrm{F}$ & $P$ \\
\hline Tamaño de individuo & 1,38 & 11,84 & $\mathbf{0 , 0 0 1}$ & 1,38 & 14,98 & $<\mathbf{0 , 0 0 1}$ \\
Número de hojas & 1,38 & 0,20 & 0,653 & 1,38 & 539,56 & $<\mathbf{0 . 0 0 0 1}$ \\
Área foliar & 1,38 & 4,99 & $\mathbf{0 , 0 3 1}$ & 1,38 & 7,54 & $\mathbf{0 , 0 0 9}$ \\
Área foliar específica & 1,38 & 1,21 & 0,28 & 1,38 & 6,95 & $\mathbf{0 , 0 1 2}$ \\
Índice de circularidad & 1,38 & 16,60 & $<\mathbf{0 , 0 0 1}$ & 1,38 & 2035,73 & $<\mathbf{0 , 0 0 0 1}$ \\
Densidad foliar & 1,38 & 5,74 & $\mathbf{0 , 0 2}$ & 1,38 & 39,98 & $<\mathbf{0 , 0 0 1}$ \\
Densidad de pubescencia & - & - & - & 1,38 & 96,73 & $<\mathbf{0 , 0 0 0 1}$ \\
Longitud de pubescencia & - & - & - & 1,38 & 331,11 & $<\mathbf{0 , 0 0 0 1}$ \\
\hline
\end{tabular}


Variación morfo-fisiológica en plantas de alta montaña: MolinA-Montenegro, M. \& L. CAVIERES

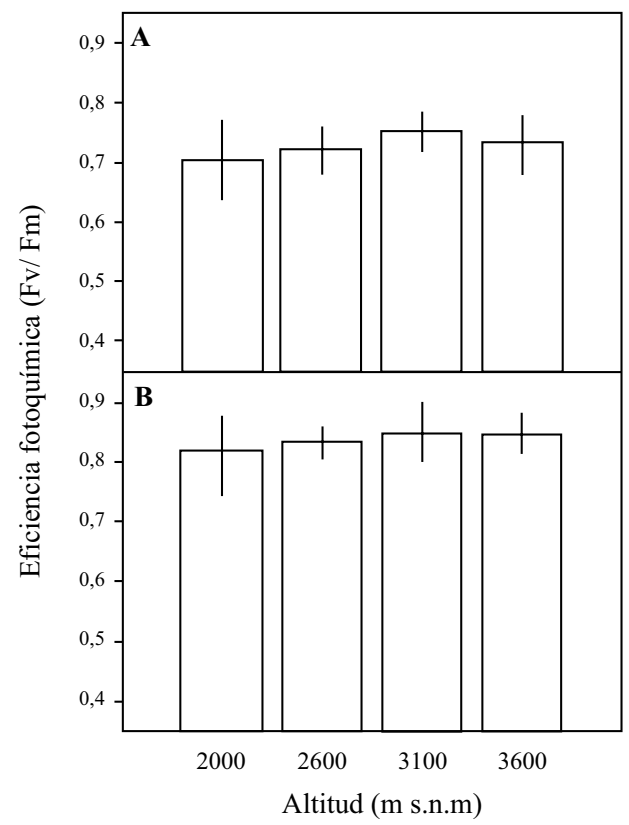

FIGURA 1. Eficiencia fotoquímica del fotosistema II (Fv/ Fm) registrada en individuos de Taraxacum officinale (A) y Phacelia secunda (B) creciendo sobre un gradiente altitudinal en Los Andes de Chile central. No se registraron diferencias a lo largo del gradiente altitudinal ni para $T$. officinale ni para $P$. secunda $\left(\mathrm{F}_{36,3}=1,66 ; \mathrm{p}=0,19\right.$ y $\mathrm{F}_{36,3}=1,89 ; \mathrm{p}=0,15$, respectivamente).

FIGURE 1. Photochemical efficiency of photosystem II (Fv/ Fm) registered in individuals of Taraxacum officinale (A) and Phacelia secunda (B) growing on altitudinal gradient in Los Andes of central Chile. Not differences were found in T. officinale and P. secunda growing on the altitudinal gradient $\left(\mathrm{F}_{36,3}=1.66 ; \mathrm{p}=0.19 \mathrm{y} \mathrm{F}_{36,3}=1.89 ; \mathrm{p}=0.15\right.$, respectively $)$.

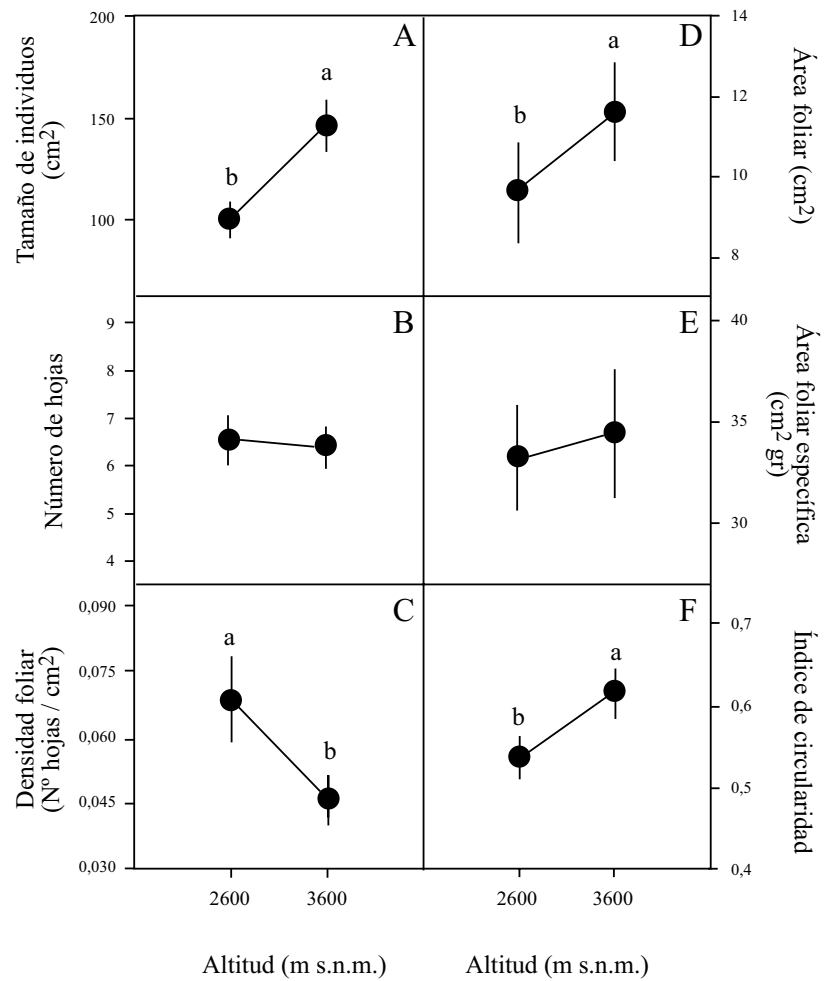

FiguRA 2. Diferentes atributos morfológicos medidos en individuos de Taraxacum officinale creciendo a los 2.600 y $3.600 \mathrm{~m}$, en Los Andes de Chile central. Letras diferentes corresponden a diferencias significativas entre altitudes (a posteriori Tukey test $\alpha=0,05$ ).

FigURE 2. Different morphological traits in Taraxacum officinale growing at 2,600 and 3,600 m, in Los Andes of central Chile. Significant differences between altitudes are denoted with different letters (a posteriori Tukey test $\alpha=0.05$ ). 
Los individuos de Phacelia secunda, creciendo a los $3.600 \mathrm{~m}$, presentaron significativamente individuos de mayor tamaño, mayor número de hojas promedio por individuo, mayor densidad foliar, hojas con un mayor índice de circularidad y pubescencia foliar de mayor longitud, en comparación a individuos de la misma especie situados a los $2.600 \mathrm{~m}$ (Fig. 3, Tabla I). Aquellos individuos distribuidos en el piso altitudinal inferior presentaron significativamente una mayor área foliar, mayor área foliar específica y una mayor densidad de pubescencia foliar (Fig. 3, Tabla I).

Por otro lado, al comparar los ángulos foliares no se registraron diferencias significativas $\left(\mathrm{F}_{38,1}=3,1 ; \mathrm{p}=0,08\right)$ entre los individuos de $T$. officinale provenientes de ambas altitudes. Sin embargo, al comparar los individuos de $P$. secunda se evidenció un aumento significativo $\left(\mathrm{F}_{38,1}=95,7\right.$; $\mathrm{p}<0,0001)$ en los ángulos foliares en aquellos individuos creciendo a los $2.600 \mathrm{~m}$ (Fig. 4).
Pigmentos Fotoprotectores

El contenido absoluto de pigmentos implicados en el ciclo de las xantófilas fue significativamente mayor $\left(\mathrm{F}_{12,1}=\right.$ 245,1; $<<0,0001$ ) en los individuos de Taraxacum officinale creciendo a los $3.600 \mathrm{~m}$, en comparación con aquéllos del piso altitudinal inferior (Fig. 5). Por otra parte, entre los individuos de Phacelia secunda provenientes de ambas altitudes no se encontraron diferencias significativas $\left(F_{12,1}=0,76 ; p=0,39\right)$ al comparar la concentración absoluta de pigmentos implicados en el ciclo de las xantófilas (Fig. 5). Luego, al comparar el estado de desepoxidación de los pigmentos fotoprotectores se evidenció que, tanto en $T$. officinale como en $P$. secunda, aquellos individuos provenientes de los 3.600 $\mathrm{m}$ presentaron significativamente $\left(\mathrm{F}_{12,1}=242,10 ; \mathrm{p}<0,0001 \mathrm{y}\right.$ $\mathrm{F}_{12,1}=163,85 ; \mathrm{p}<0,0005$, respectivamente) mayores niveles en comparación a individuos de la misma especie creciendo a los $2.600 \mathrm{~m}$ (Fig. 5).

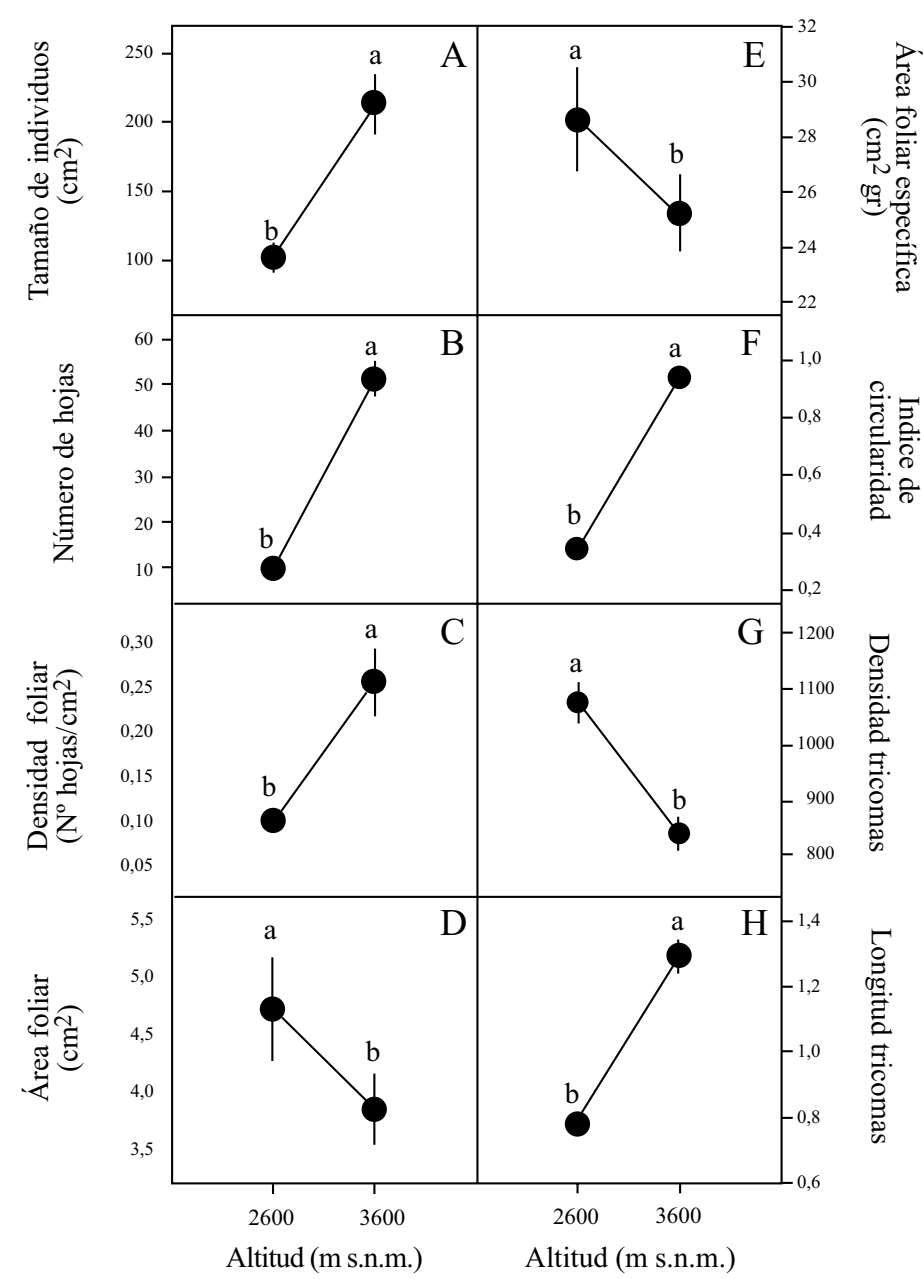

Figura 3. Diferentes atributos morfológicos medidos en individuos de Phacelia secunda creciendo a los 2.600 y $3.600 \mathrm{~m}$, en Los Andes de Chile central. Letras diferentes corresponden a diferencias significativas entre altitudes (a posteriori Tukey test $\alpha=0,05$ ).

FIgURE 3. Different morphological traits in Phacelia secunda growing at 2,600 and 3,600 m, in Los Andes of central Chile. Significant differences between altitudes are denoted with different letters (a posteriori Tukey test $\alpha=0.05$ ). 
Variación morfo-fisiológica en plantas de alta montaña: MolinA-Montenegro, M. \& L. CAVIERES

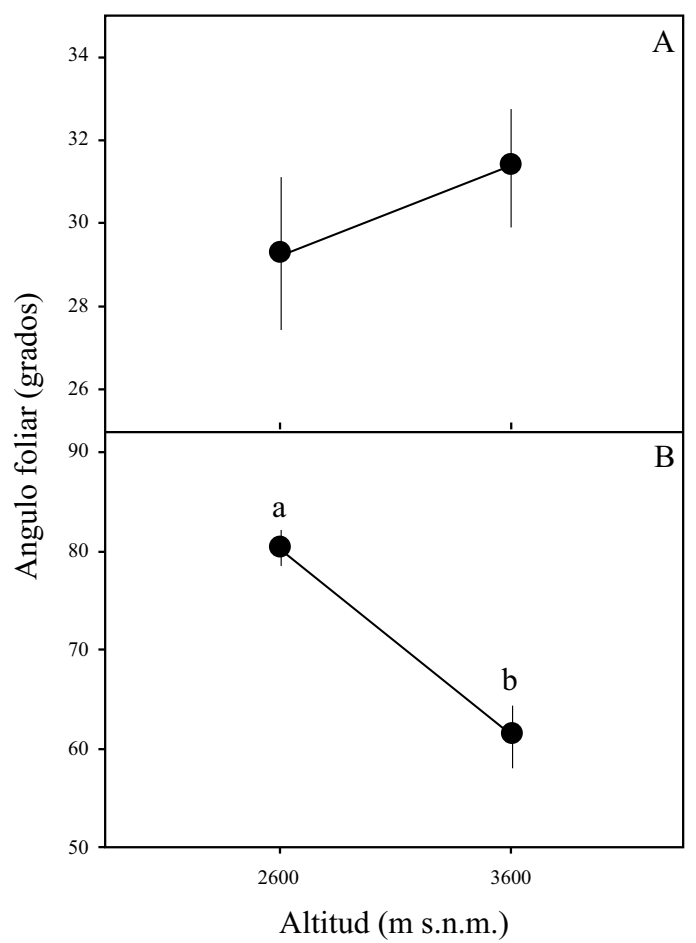

FIGURA 4. Variación de los ángulos foliares registrados en individuos de Taraxacum officinale (A) y Phacelia secunda (B) creciendo a los 2.600 y $3.600 \mathrm{~m}$, en Los Andes de Chile central. Letras diferentes corresponden a diferencias significativas entre altitudes (a posteriori Tukey test $\alpha=0,05$ ).

FIGURE 4. Leaf angles variation in individuals of Taraxacum officinale (A) and Phacelia secunda (B) growing at 2,600 and 3,600 m, in Los Andes of central Chile. Significant differences between altitudes are denoted with different letters (a posteriori Tukey test $\alpha=0.05$ ).

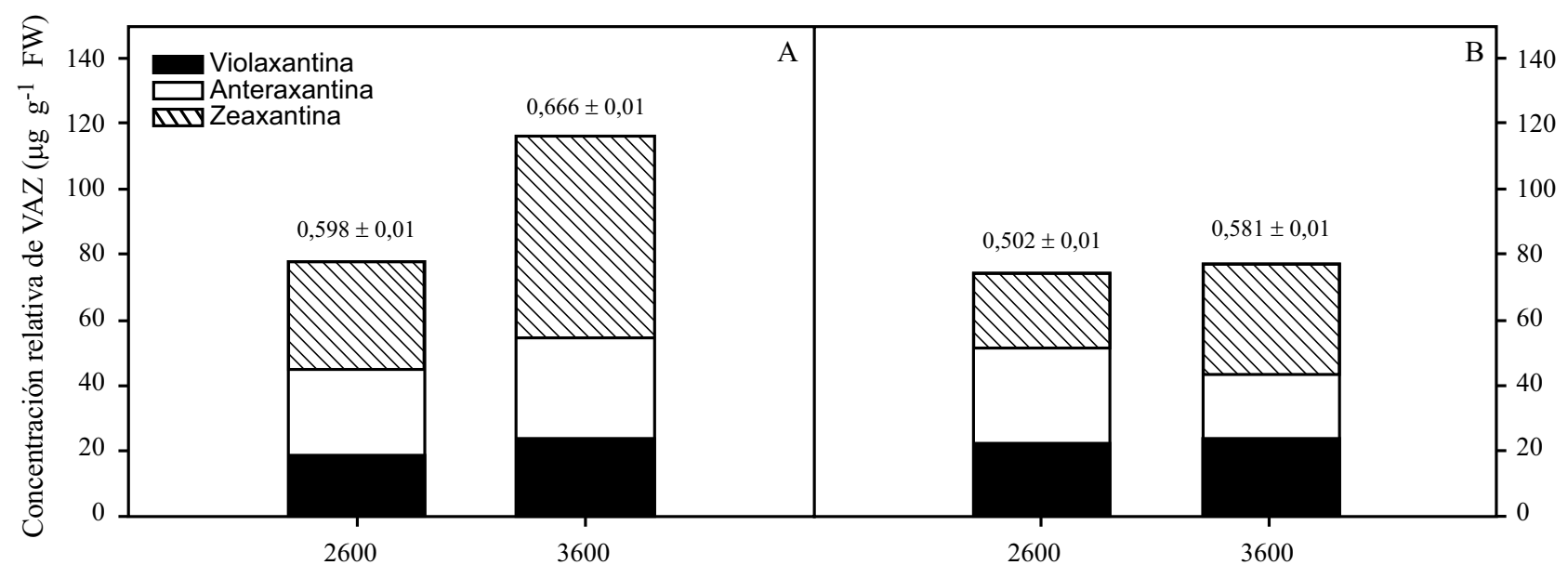

Altitud (msnm)

Altitud (msnm)

FIGURA 5. Variación de los contenidos de pigmentos implicados en el ciclo de las xantófilas en individuos de Taraxacum officinale (A) y Phacelia secunda (B), creciendo a los 2.600 y 3.600 m, en Los Andes de Chile central. Los valores sobre cada barra corresponden al estado de desepoxidación ( $\pm 2 \mathrm{EE})$.

FIGURE 5. Variation in the amount of pigments involved in xanthophyll cycle in individuals of Taraxacum officinale (A) and Phacelia secunda (B), growing at 2,600 and 3,600 m, in Los Andes of central Chile. Values on each bar denote the desepoxidation status ( $\pm 2 \mathrm{EE}$ ). 


\section{DISCUSIÓN}

La altitud representa un gradiente ambiental complejo, donde la temperatura, radiación solar y condiciones del suelo (e.g., humedad y nutrientes) varían significativamente. La temperatura es fuertemente influenciada por la altitud, menores altitudes tendrán mayores temperaturas y por lo tanto un aumento en la actividad metabólica, crecimiento celular, tasa fotosintética y por ende mayor crecimiento en tamaño (Körner et al. 1989, 2003). No obstante, en nuestro trabajo encontramos que el tamaño promedio de ambas especies aumentó de manera significativa con la altitud, sugiriendo que las condiciones ambientales no serían necesariamente limitantes para el desarrollo celular y por ende del crecimiento vegetativo. Resultados similares fueron documentados por Cavieres (2000), quien demostró que la especie $P$. secunda a medida que aumenta en altitud presenta un crecimiento más postrado y con individuos de mayor tamaño.

Por otro lado, los individuos de T. officinale presentaron diferencias en su morfología en cuatro de los seis atributos morfológicos evaluados, mientras que los individuos de $P$. secunda mostraron una gran variación de todos los atributos morfológicos con la altitud. La variación en la circularidad foliar ha sido descrita previamente como el resultado de adaptaciones ambientales locales (Gianoli 2004). Un aumento en la circularidad foliar disminuye el perímetro de contacto con el aire circundante, lo cual repercute en un menor intercambio calórico con el ambiente. Así, en las poblaciones de mayor altitud la presencia de hojas más circulares podría significar una menor pérdida de calor, debido a que se generaría un gradiente térmico mayor desde el centro de la hoja hacia la periferia, aumentando el desacople térmico entre la hoja y el ambiente (Nobel 2005).

Se ha documentado que el crecimiento horizontal y más cercano al sustrato es una estrategia para aumentar la eficiencia en la captación de calor y regulación de la energía térmica, de modo que los tejidos vegetales pueden estar a varios grados por sobre la temperatura ambiental (Körner 2003). Considerando que los individuos de $P$. secunda a los 3.600 no sólo presentan un crecimiento postrado sino que un mayor tamaño y hojas más circulares, sugiere que la morfología de esta especie podría actuar como una eficiente trampa de calor manteniendo sus hojas a temperaturas por sobre la del ambiente. Por otro lado, la alta densidad de hojas pubescentes y menores ángulos foliares podrían contribuir al autosombreamiento de la planta, evitando que los individuos de $P$. secunda que habitan a mayores elevaciones presenten signos de fotoinhibición. Las variaciones en la orientación $\mathrm{y}$ en los ángulos foliares han sido documentadas como estrategias efectivas para evitar la fotoinhibición, en la cual mayores ángulos foliares evitarían que la radiación solar incidiera de manera directa (Ehleringer \& Forseth 1980,
Kao \& Forseth 1992, Muraoka et al. 1998, Ikeda \& Matsuda 2002). Sin embargo, los individuos de $P$. secunda creciendo en condiciones de mayor radiación lumínica presentaron menores ángulos foliares. Una hipótesis alternativa para este resultado es que las hojas más expuestas a la radiación actuarían como pantalla protectora, donde unas pocas hojas podrían "sacrificarse" por el individuo completo. No obstante, estudios más detallados debiesen ser realizados para respaldar esta hipótesis.

La presencia de la "capa límite de aire en las hojas" ha sido sugerida como una componente reguladora de la temperatura foliar (Rundel et al. 1994, MolinaMontenegro 2008). La presencia de pubescencia foliar es una característica común en plantas de ambientes con alta radiación o bajas temperaturas, debido a que al modificar el grosor de la capa límite, afectarían en última instancia al balance energético del individuo (Chandra 2004). $P$. secunda presenta una variación tanto en la longitud como en la densidad de su pubescencia foliar con la altitud. Aquellos individuos presentes en los $3.600 \mathrm{~m}$ poseen estructuras de mayor longitud pero en menor densidad comparados con individuos de la misma especie de los $2.600 \mathrm{~m}$. El aumento altitudinal de la pubescencia también ha sido documentado previamente para algunas especies de amplio rango de distribución en zonas alpinas (Meinzer et al. 1985, MolinaMontenegro 2008). La mayor longitud de la pubescencia con la altitud permite aumentar el grosor de la "capa límite" disminuyendo la pérdida de calor por convección (Meinzer \& Goldstein 1985). Esto le permite a la planta mantener un balance térmico favorable en comparación a las frías condiciones del aire que las rodea. De un modo similar, una mayor longitud de la pubescencia foliar permite crear una película sobre la epidermis que restringe la intensidad de la incidencia de fotones (Skaltsa et al. 1994). Considerando que la intensidad de la radiación aumenta con la altitud (Körner 2003), una mayor pubescencia foliar también cumpliría la función de proteger contra el exceso de radiación del ambiente.

La baja variación morfológica en los individuos de $T$. officinale con la altitud podría sugerir la presencia de un eficiente sistema fisiológico para evitar la fotoinhibición por alta radiación y bajas temperaturas. En nuestro estudio se evidenció, que de manera significativa, aquellos individuos de $T$. officinale que crecen a los $3.600 \mathrm{~m}$ presentaron mayores concentraciones de pigmentos disipadores de energía en comparación a aquéllos presentes a los 2.600 $\mathrm{m}$. Por otra parte, la concentración de pigmentos no fue diferente en los individuos de $P$. secunda creciendo en ambas altitudes. Estos resultados estarían sugiriendo que mientras $T$. officinale posee una estrategia principalmente fisiológica para evitar fotoinhibirse a mayores elevaciones, $P$. secunda lo hace a través de estrategias principalmente morfológicas. Una gran cantidad de estudios (Adams \& Demming-Adams 1992, Demming-Adams \& Adams 1996, 
Thiele et al. 1996, García-Plazaola et al. 2007) han descrito que la concentración de pigmentos implicados en el ciclo de las xantófilas aumenta en condiciones de alta radiación y/o bajas temperaturas y que sería uno de los principales mecanismos para evitar la fotoinhibición en ambientes de alta-montaña (Streb et al. 1998, Körner 2003, Williams et al. 2003). Un estudio realizado con especies arbustivas en una zona mediterránea demostró que las bajas temperaturas de invierno inducen un aumento en el contenido de pigmentos implicados en el ciclo de las xantófilas, lo cual les permite disipar la energía excesiva, alcanzando concentraciones similares a las encontradas en este estudio (Kyparissis et al. 2000). De manera similar, Williams et al. (2003) demostraron que las bajas temperaturas y la alta radiación lumínica aumentan los niveles de zeaxantina en plantas de zonas alpinas como estrategia para disipar la energía en exceso evitando la fotoinhibición. De esta manera, especies provenientes de zonas de alta-montaña mantendrían mayores niveles totales de pigmentos y específicamente de zeaxantina como una estrategia fotoprotectora.

Por otro lado, el estado de desepoxidación de ambas especies fue mayor en aquellos individuos creciendo a los $3.600 \mathrm{~m}$, lo cual indicaría que a mayor elevación ambas especies estarían disipando la radiación solar en exceso como calor. Esto último estaría indicando que si bien los individuos de $P$. secunda, provenientes de los $3.600 \mathrm{~m}$, no poseen mayor concentración de pigmentos que aquéllos de los $2.600 \mathrm{~m}$, una mayor proporción de los pigmentos estaría en estado de zeaxantina. Se ha documentado que plantas distribuidas en ambientes con altos niveles de radiación o heterogéneos lumínicamente presentarían mayores niveles de desepoxidación (Adams \& Demming-Adams 1992, Munné-Bosch \& Peñuelas 2003). Por ejemplo, MunnéBosch \& Alegre (2000) demostraron que una condición de alta radiación lumínica estimularía un aumento en el estado de desepoxidación como mecanismo fotoprotector del fotosistema II. De manera similar, Munné-Bosch \& Peñuelas (2003) sugieren que unas pocas moléculas de zeaxantina por centro de reacción del fotosistema II en combinación con un gradiente de acidez a través de la membrana tilacoidal serían requeridos para una eficiente disipación térmica. De esta manera, una elevada tasa de desepoxidación más que la acumulación de pigmentos sería necesaria para una eficiente disipación térmica de la energía en exceso. Considerando que el sitio de mayor altitud utilizado en este estudio está sujeto a condiciones lumínicas más intensas y eventos más frecuentes de bajas temperaturas, la presencia de altos niveles de zeaxantina junto a altos niveles de desepoxidación podrían proteger a las plantas de los riesgos de fotoinhibición.

El éxito de establecimiento de una especie en sitios donde las condiciones ambientales son propicias para la fotoinhibición dependerá en gran medida de la presencia de atributos morfo-fisiológicos que posea (Körner 2003).
Los ambientes de alta-montaña han sido sugeridos como zonas poco propicias para eventos de colonización y establecimiento (Cavieres et al. 2005). No obstante, T. officinale y P. secunda son dos ejemplos de especies que presentan diversas estrategias para lidiar de manera eficiente con las condiciones ambientales que puedan encontrar sobre su gradiente de distribución altitudinal. De esta manera, la presencia de una determinada especie en la zona de la alta-montaña dependerá en gran medida de las estrategias particulares que posea cada una de ellas para evitar la fotoinhibición por alta radiación y bajas temperaturas típica de las zonas de la alta-montaña de Chile central.

\section{AGRADECIMIENTOS}

MAM-M agradece a la beca doctoral CONICYT y a los proyectos FONDECYT 1060710 y P02-051-F ICM. Este trabajo es parte de las actividades de investigación del Instituto de Ecología y Biodiversidad (IEB).

\section{BIBLIOGRAFÍA}

Adams, W.W. \& B. Demming-Adams. 1992. Operation of the xanthophyll cycle in higher plants in response to diurnal changes in incident sunlight. Planta 186: 390-398.

CaAsi-Lit, M., M.I. Whitecross, N. Nayudu. \& G.J. Tanner. 1997. UV-B irradiation induces differential leaf damage ultrastructural changes and accumulation of specific phenolic compounds in rice cultivars. Journal of Plant Physiology 24: 261-274.

Cavieres, L.A. 2000. Variación morfológica de Phacelia secunda J.F.Gmel. (Hydrophyllaceae) a lo largo de un gradiente altitudinal en Chile central. Gayana Botánica 57: 89-96.

Cavieres, L.A. \& M.T.K. Arroyo. 1999. Tasa de enfriamiento adiabático del aire en el valle del río Molina, Provincia de Santiago, Chile central ( $\left.33^{\circ} \mathrm{S}\right)$. Revista Geográfica de Chile Terra Australis 44: 79-86.

Cavieres, L.A., C.L. Quiroz, M.A. Molina-Montenegro, A.A. MuÑoz \& A. PAuchard. 2005. Nurse effect of the native cushion Azorella monantha on the invasive non-native Taraxacum officinale in the high-Andes of central Chile. Perspectives in Plant Ecology, Evolution and Systematics 7: $217-226$

Cavieres, L.A., C.L. Quiroz \& M.A. Molina-Montenegro. 2008. Facilitation of the non-native Taraxacum officinale by native nurse cushion species in the high Andes of central Chile: are there differences between nurses? Functional Ecology 22: 148-156.

Chandra, S. 2004. Effects of altitude on energy exchange characteristics of some alpine medicinal crops from central Himalayas. Journal of Agronomy \& Crops Science 190: 13-20.

Cui, X., Y. Tang, S. Gu, S. Nishimura, S. Shi \& X. Zhao. 2003. Photosynthetic depression in relation to plant architecture in two alpine herbaceous species. Environmental and Experimental Botany 50: 125-135.

Deginani, N.B. 1982. Revisión de las especies argentinas del género Phacelia (Hydrophyllaceae). Darwiniana 24: 405-496. 
Demming-Adams, B. \& W. Adams. 1996. The role of xanthophyll cycle carotenoids in the protection of photosynthesis. Trends in Plant Science 1: 21-26.

EhLeringer, J. \& I. Forseth. 1980. Solar tracking by plants. Science 1094-1098.

Galen, C. 2006. Solar furnaces or swamp coolers: costs and benefits of water use by solar-tracking flowers of the alpine snow buttercup, Ranunculus adoneus. Oecologia 48: 195-201.

García-Plazaola, J.I., S. Matsubara \& C.B. Osmond. 2007. The lutein epoxide cycle in higher plants: its relationships to other xanthophyll cycles and possible functions. Functional Plant Biology 34: 759-773.

Germino, M.J. \& W.K. Smith. 2000. High resistence to lowtemperature photoinhibition in two alpine, snowbank species. Physiologia Plantarum 110: 89-95.

Gianoli, E. 2004. Plasticity of traits of correlations in two populations of Convolvulus arvensis (Convolvulaceae) differing in environmental heterogeneity. International Journal of Plant Sciences 165: 825-832.

GiLmore, A.M. 1997. Mechanistic role of Xanthophyll-dependent photoprotection in higher plant chloroplasts and leaves. Physiologia Plantarum 99: 197-209.

Huner, N.P.A., G. ÖQuist \& F. SARHAn. 1998. Energy balance and acclimation to light and cold. Trends in Plant Science 6: 224-230.

IKedA, T. \& R. MAtsudA. 2002. Effects on soyabean leaflet inclination on some factors related to photosynthesis. Journal of Agriculture Science 138: 367-373.

KaO, W.Y. \& Y.N. Forseth. 1992. Responses of gas exchange and phototropic leaf orientation in soybean to soil water availability, leaf water potential, air temperature and photosynthetic photon flux. Environmental and Experimental Botany 32: 343-349.

KöRner, C. 2003. Alpine Plant Life (2nd ed). Springer, Berlin. $344 \mathrm{pp}$.

KöRner, C. \& W. LARChER. 1988. Plant life in cold climates. In: S.F. Long \& F.I. Woodward (eds.), Plants and Temperature, pp. 25-57. Cambridge University Press, Cambridge.

Körner, C., M. Neumayer, S. Peláez Menéndez-Riedl \& A. SMEETS-SCHEEL. 1989. Functional morphology of mountain plants. Flora 182: 353-383.

Kyparissis，A., P. Drilias \& Y. Manetas. 2000. Seasonal fluctuations in photoprotective (xanthophyll cycle) and photoselective (chlorophylls) capacity in eight Mediterranean plant species belonging to two different growth forms. Australian Journal of Plant Physiology 27: 265-272.

LonG, S.P., S. Humphries \& P.G. FAlKowsKi. 1994. Photoinhibition of photosynthesis in nature. Annual Review Plant Physiology Plant Molecular Biology 45: 633-662.

Manuel, N., G. Cornic, S. Aubert, P. Choler, R. Bligny \& U. Heber. 1999. Protection against photoinhibition in the alpine plant Geum montanum. Oecologia 119: 149-158.

Maxwell, A. \& G.N. Johnson. 2000. Chlorophyll fluorescence - a practical guide. Journal of Experimental Botany 345: 659-668.

Meinzer, F. \& G. Goldstein. 1985. Some consequences of leaf pubescence in the Andean giant rosette plant Espeletia timotensis. Ecology 66: 512-520.

Meinzer, F., G. Goldstein \& P. Rundel. 1985. Morphological changes along an altitude gradient and their consequences for an Andean giant rosette plant. Oecologia 65: 278-283.

Molina-Montenegro, M.A. 2008. Variación de la pubescencia foliar en plantas y sus implicaciones funcionales a lo largo de gradientes altitudinales. Ecosistemas 17: 146-154.

Molina-Montenegro, M.A., R. Briones \& L.A. Cavieres. 2009. Does global warming induce segregation among alien and native beetle species in a mountain-top? Ecological Research 24: 31-36.

Munné-Bosch, S. \& L. Alegre. 2000. The significance of $\beta$ carotene, $\alpha$-tocopherol and xanthophyll cycle in droughted Melissa officinalis plants. Australian Journal of Plant Physiology 27: 139-146.

Munné-Bosch, S. \& J. Peñuelas. 2003. Photo- and antioxidative protection during summer leaf senescence in Pistacia lentiscus L. grown under Mediterranean fields conditions. Annals of Botany 92: 385-391.

Muraoka, H., A. Takenaka, Y. Tang, H. Koizumi \& I. Washitani. 1998. Flexible leaf orientations of Arisaema heterophyllum maximize light capture in a forest understory and avoid excess irradiance at a deforested site. Annals of Botany 82: 297-307.

NiYogI, K.K. 1999. Photoprotection revisited: genetic and molecular approaches. Annual Review Plant Physiology and Plant Molecular Biology 50: 333-359.

NiYogi, K.K. 2000. Safety valves for photosynthesis. Current Opinion in Plant Biology 3: 455-460.

NoBel, P.S. 2005. Physicochemical and environmental plant physiology. Academic Press, New York. 582 pp.

Öncel, I., E. Yurdakulol, Y. Keles, L. Kurt \& A. Yildiz. 2004. Role of antioxidant defense system and biochemical adaptation on stress tolerance of high mountain and steppe plants. Acta Oecologica 26: 211-218.

Osmond, C.B. 1994. What is photoinhibition? Some insights from comparisons of shade and sun plants. In: N.R. Baker \& J.R. Bowyer (eds.), Photoinhibition of photosynthesis: from molecular mechanisms to the field: 1-24. BIOS Scientific Publishers Limited, Oxford, United Kingdom.

Pérez-Torres, E., A. García, J. Dinamarca, M. Alberdi, A. GutiérRez, M. Gidekel, A.G. Ivanov, N.P.A. HüNer, L.J. Corcuera \& L.A. Bravo. 2004. The role of photochemical quenching and antioxidants in photoprotection of Deschampsia antarctica. Functional Plant Biology 31: 731-741.

Pittermann, J. \& R.F. Sage. 2000. Photosynthetic performance at low temperature of Bouteloua gracilis Lag., a highaltitude C4 grass from the Rocky Mountains, USA. Plant Cell and Environment 23: 811-823.

Rundel, P., A. Smith, \& F. Meinzer. 1994. Tropical Alpine Environments. Cambridge University Press, UK. 392 pp.

SAntibÁÑEz, F. \& J.M. URibe. 1990. Atlas agroclimático de Chile. Regiones VIII y IX. Ediciones Universidad de Chile, Santiago, Chile. 99 pp.

Skaltsa, H., E. Verykokidou, C. Harvala, G. Karabourniotis \& Y. MANETAS. 1994. UV-B protective potential and flavonoid content of leaf hair of Quercus ilex. Phytochemistry 37: 987-990.

SOKAL R. \& F.J. RoHLF. 1995. Biometry: the principles and practice of statistics in biological research. Freeman Company, New York. 887 pp.

Streb, P., S. Aubert, E. Gout \& R. Bligny. 2003a. Reversibility of cold- and light-stress tolerance and accompanying changes of metabolite and antioxidant levels in the two high mountain plant species Soldanella alpina and Ranunculus glacialis. Journal of Experimental Botany 54: 405-418.

Streb, P., S. Aubert, E. Gout \& R. Bligny. 2003b. Cold- and light- induced changes of metabolite and antioxidant levels in two high mountain plant species Soldanella 
Variación morfo-fisiológica en plantas de alta montaña: MolinA-Montenegro, M. \& L. CAVIERES

alpina and Ranunculus glacialis and a lowland species Pisum sativum. Physiologia Plantarum 118: 96-104.

Streb, P., J. Feierabend \& R. Bligny. 1997. Resistance to fotoinhibition of photosystem II and catalase and antioxidative protection in high mountain plants. Plant, Cell and Environment 20: 1030-1040.

Streb, P., J. Feierabend \& R. Bligny. 1998. Divergent strategies of photoprotection in high-mountain plants. Planta 207: 313-324.

Thiele, A., K. Schirwitz, K. Winter \& G.H. Krause. 1996. Increased xanthophyll cycle activity and reduced D1 protein inactivation related to photoinhibition in two plant systems acclimated to excess light. Plant Science 115: 237-250.
WILDI, B. \& C. LÜTZ. 1996. Antioxidant composition of selected high alpine plant species from different altitudes. Plant, Cell and Environment 19: 138-146.

Williams, E.L., M.J. Hovenden \& D.C. Clase. 2003. Strategies of Light energy utilisation, dissipation and attenuation in six co-occurring alpine heath species in Tasmania. Functional Plant Biology 30: 1205-1218.

Zuloaga, F.O., O. Morrone \& M.J. Belgrano. 2008. Catálogo de las Plantas Vasculares del Cono Sur (Argentina, Sur de Brasil, Chile, Paraguay y Uruguay). Monograph in Systematic Botany from Missouri Botanical Garden 107. $3348 \mathrm{pp}$. 